\title{
Fracasso Escolar na Educação Básica: Revisão Sistemática da Literatura
}

\author{
Fernanda Aparecida Szareski Pezzi ${ }^{1}$ \\ Sociedade Educacional Três de Maio, Três de Maio, RS, Brasil \\ Angela Helena Marin \\ Centro de Ciências da Saúde da Universidade do Vale do Rio dos Sinos, \\ São Leopoldo, RS, Brasil
}

\begin{abstract}
Resumo
O objetivo deste estudo foi realizar uma revisão sistemática da literatura sobre o fracasso escolar na educação básica, considerando o período de janeiro de 2009 a dezembro de 2014, a fim de analisar o ano e o país da publicação, o foco do estudo, o conceito de fracasso escolar adotado, os aspectos metodológicos e os principais resultados. Foram selecionados 44 artigos das bases de dados: Academic Search Complete, Literatura Latino-Americana em Ciências da Saúde (LILACS), PsyINFO, Scientific Electronic Library Online (SciELO) e Web of Science. Os artigos derivaram de 18 países e apresentaram foco e conceitos diferentes sobre o fracasso escolar. Quanto aos aspectos metodológicos,a abordagem quantitativa foi a mais utilizada, os alunos foram os principais participantes das pesquisas e diversos instrumentos foram utilizados, permitindo a triangulação de dados. De modo geral, a revisão de literatura realizada possibilitou conhecer a forma como o fracasso escolar vem sendo estudado e compreendido nas pesquisas atuais, representando uma fonte importante de consulta para profissionais da área da psicologia e da educação.
\end{abstract}

Palavras-chave: Fracasso escolar, reprovação escolar, educação básica.

\section{School Failure in Elementary School: A Systematic Literature Review}

\begin{abstract}
This study aimed to conduct a systematic literature review on school failure in elementary school, from January 2009 to December 2014, in order to analyze the year and country of publication, the focus of the study, the concept of school failure adopted, the methodological aspects, and the main results. A number of 44 articles were selected, from the following database: Academic Search Complete, Literatura LatinoAmericana em Ciências da Saúde (LILACS), PsycINFO, Scientific Electronic Library Online (SciELO) and Web of Science. The articles came from 18 different countries and presented different concepts and focus on school failure. In terms of methodological issues, most of the articles used a quantitative approach, most of them with the participation of students and the use of different instruments allowing data triangulation. Overall, the literature review conducted helped to understand how school failure has
\end{abstract}

Endereço para correspondência: Sociedade Educacional Três de Maio - Setrem, Avenida Santa Rosa, 2405 - Centro Três de Maio - RS, CEP 98910-000. E-mail: nandaszareski@yahoo.com.br

Artigo derivado da dissertação de mestrado de Fernanda Aparecida Szareski Pezzi, realizada sob a orientação de Angela Helena Marin, apresentada no Programa de Pós-Graduação em Psicologia da Universidade do Vale do Rio dos Sinos - São Leopoldo - RS.

Apoio financeiro: Conselho Nacional de Desenvolvimento Científico e Tecnológico (CNPq) e Coordenação de Aperfeiçoamento de Pessoal de Nível Superior (CAPES). 
been studied and understood in current researches representing a valuable resource for psychology and education professionals.

Keywords: School failure, school failure, elementary school.

\section{Fracaso Escolar en la Educación Básica: Revisión Sistemática de la Literatura}

\section{Resumen}

El objetivo de este estudio fue realizar una revisión sistemática de la literatura sobre el fracaso escolar en la educación básica, teniendo en cuenta el período de enero de 2009 a diciembre de 2014 con el fin de analizar el año y el país de publicación, el foco del estudio, el concepto de fracaso escolar adoptado, los aspectos metodológicos y los principales resultados. Se seleccionaron 44 artículos de las bases de datos: Academic Search Complete, Literatura Latino-Americana em Ciências da Saúde (LILACS), PsyINFO, Scientific Electronic Library Online (SciELO) y Web of Science. Los artículos derivaron de 18 países y presentaron enfoques y conceptos diversos respecto al fracaso escolar. En cuanto a los aspectos metodológicos, el enfoque cuantitativo fue el más utilizado y hubo una mayor participación de los estudiantes y el uso de diferentes instrumentos, que permitieron la triangulación de datos. En general, la revisión bibliográfica realizada ha permitido conocer la forma en que se ha estudiado y comprendido el fracaso escolar en la investigación actual, lo que representan una fuente importante de consulta para los profesionales de la psicología y de la educación.

Palabras clave: Fracaso escolar, desaprobación escolar, educación básica.

A temática do fracasso escolar tem sido mundialmente estudada (Bastos, Fernandes, \& Passos, 2009; Hancer, 2012; Kamal \& Bener, 2009; Lagana-Riordan et al., 2011; Marshall, 2003; Ullastres, 2003) e a expressão tem sido utilizada para se referir a uma série de fenômenos educacionais, como: reprovação, baixo rendimento, distorção idade-série/ano e dificuldades de aprendizagem (Zago, 2011). Embora as estatísticas revelem uma redução no fracasso escolar ao longo dos anos (Instituto Brasileiro de Geografia e Estatística [IBGE], 2010; Organização para a Cooperação e o Desenvolvimento Econômico [OCDE], 2010), um em cada cinco alunos dos mais de trinta países que compõem a OCDE (1998) não atinge o nível básico de formação (OCDE, 2012). No Brasil, as taxas de distorção idade-série/ano (23,6\%), reprovação $(10,3 \%)$ e abandono escolar $(3,1 \%)$ no ensino fundamental também continuam sendo significativas e preocupantes (IBGE, 2010). A permanência da alta incidência do fracasso escolar na atualidade é um desafio para a qualidade da educação e impõe dificuldades individuais, familiares e sociais.
A Organização das Nações Unidas para a Educação, Ciência e Cultura - Unesco tem articulado ações internacionais para garantir o direito à educação, já assegurado na Declaração Universal dos Direitos Humanos (Organização das Nações Unidas [ONU], 1948) e reafirmado em documentos como a Declaração Mundial sobre Educação para Todos (1990) e no Acordo de Dakar (2000). Neste último, 164 países, incluindo o Brasil, se comprometeram a qualificar a educação, garantindo o acesso universal de todas as pessoas à escola, a erradicação do analfabetismo e a atenção às necessidades básicas de aprendizagem. Entretanto, os dados mundiais têm apontado para a falta de foco na qualidade da educação, bem como o insucesso em alcançar as crianças que se encontram à margem do sistema escolar (57 milhões de crianças não têm acesso à escola), e também aquelas que estão na escola, mas não conseguem desenvolver as habilidades básicas de aprendizagem (250 milhões de crianças), não atingindo as aptidões necessárias para conseguirem bons empregos e terem realização pessoal e profissional (Unesco, 2014). 
No projeto lançado pela OCDE (2010) destacou-se que o fracasso escolar penaliza uma criança por toda sua vida, uma vez que ela deixa de estar em nível de igualdade com as demais, acarretando, futuramente, empregabilidade em ocupações com salários mais baixos e, consequente aposentadoria inferior. Neste sentido, as consequências do fracasso escolar também são relevantes para a sociedade, pois podem ocasionar o aumento da criminalidade e a necessidade de gastos adicionais de saúde e de assistência social (OCDE, 2010).

Faz-se importante discutir sobre o termo fracasso escolar, que pode induzir a três ideias equivocadas, segundo Marchesi e Pérez (2004). A primeira se refere ao entendimento de que o aluno não teria progredido no âmbito do conhecimento e do seu desenvolvimento pessoal e social durante a sua escolarização, o que não é necessariamente verdade. A concepção de fracasso também pode remeter à limitação da escola que não consegue atingir o seu objetivo educacional, o que pode contribuir para o afastamento dos alunos e das famílias e acentuar a dificuldade de aprendizagem. Por fim, a ideia de fracasso pode acabar concentrando no aluno o problema da reprovação, eximindo a responsabilidade de outros agentes e instituições corresponsáveis, tais como, as condições sociais, a família, a escola e o sistema educacional como um todo. $\mathrm{Na}$ busca por sanar tais equívocos, outros termos foram utilizados como "alunos com baixo rendimento escolar" ou "alunos que abandonaram o ensino fundamental". Contudo, apesar dessas tentativas, o termo fracasso escolar é amplamente difundido em todos os países e mais sintético que outras expressões, o que o torna atual e de difícil modificação (Marchesi \& Pérez, 2004). De acordo com a OCDE (1998), independentemente das diferenças no uso do termo, o fracasso escolar precisa ser considerado como um processo, que pode envolver três momentos. $\mathrm{O}$ início se dá quando o aluno tem desempenho inferior à média e precisa repetir um ano escolar; o segundo acontece no momento em que ele acaba por evadir o sistema escolar antes de completar a escolaridade obrigatória; e, por fim, quando repercute na vida profissional devido à falta de conhecimentos básicos que deveriam ter sido aprendidos na escola.

Destaca-se, assim, que o fracasso escolar não se constitui apenas como um problema educacional, mas possui repercussões individuais e sociais (Ullastres, 2003). Portanto, faz-se importante que ele seja estudado e discutido pelos profissionais da saúde, em especial os psicólogos, porque a sua manifestação e vivência podem trazer sofrimento e ter implicações clínicas importantes para os indivíduos, como, abuso de drogas, problemas de conduta (Kamal $\&$ Bener, 2009), envolvimento com a criminalidade, gravidez na adolescência (Rumberger, 2011) e, ainda, como aponta Delors (1998), suscitar situações de exclusão, considerando a lacuna que se produz no plano moral, humano e social.

Assim, ao longo dos anos distintas investigações buscaram a compreensão do fracasso escolar. Alguns estudos enfocaram os alunos e suas capacidades, a herança genética e os aspectos biológicos (Asbahr \& Lopes, 2006; Marchesi \& Pérez, 2004; Zucoloto \& Patto, 2007). Já outras, investigaram temáticas voltadas à burocratização da escola e a sua influência sobre a qualidade do trabalho docente, a distância entre as culturas escolar e popular, a inadequação do material pedagógico e a discriminação das diferenças no contexto escolar (Angelucci, Kalmus, Paparelli, \& Patto, 2004). Algumas pesquisas ainda deram ênfase aos fatores sociais e culturais (Marchesi \& Pérez, 2004). Portanto, Vieira et al. (2012) ressaltaram a importância de entender o fracasso escolar, considerando tanto as variáveis extraescolares, relacionadas às questões biológicas, sociais e emocionais dos alunos e suas famílias, como às intraescolares, arroladas ao contexto escolar, tendo em vista que todas elas interatuam na produção do mesmo.

Frente ao exposto, apresenta-se como uma necessidade a sistematização do que as pesquisas atuais (últimos seis anos) têm considerado ao trabalhar com a temática do fracasso escolar na educação básica, que hoje contempla a pré-escola, o ensino fundamental e o ensino mé- 
dio. Tal recorte de público se dá em função da obrigatoriedade do ensino, que de acordo com a Lei 12.796/2013, que altera a Lei de Diretrizes e Bases da Educação - LDB 9.394/1996, passou a contemplar o período dos quatro aos dezessete anos de idade. Com esse intuito, o presente artigo teve como objetivo realizar uma revisão sistemática da literatura sobre o fracasso escolar na educação básica, considerando o período de janeiro de 2009 a dezembro de 2014, a fim de analisar o ano e o país de origem da publicação, o foco do estudo, o conceito de fracasso escolar considerado, os aspectos metodológicos (abordagem de pesquisa, participantes e instrumentos) e seus principais resultados.

\section{Método}

Foi realizada uma revisão sistemática da literatura sobre o fracasso escolar na educação básica. As bases de dados consultadas foram: Academic Search Complete, Literatura Latino-Americana em Ciências da Saúde (LILACS), PsyINFO, Scientific Electronic Library Online (SciELO) e Web of Science. A seleção destas bases de dados para a pesquisa, ocorreu em função delas indexarem estudos sobre saúde e educação, além de serem confiáveis e reconhecidas cientificamente. A busca foi realizada através do acesso ao sítio eletrônico das próprias bases, a partir dos seguintes descritores e operadores booleanos: "fracasso escolar" $O R$ "insucesso escolar" $O R$ "reprovação escolar" e "school failure".

Foram incluídos apenas os artigos que apresentassem estudos empíricos sobre o fracasso escolar, publicados entre janeiro de 2009 e dezembro de 2014, nas línguas portuguesa, inglesa ou espanhola. Foram excluídos livros, teses, dissertações ou outras publicações que não estavam disponíveis online gratuitamente. A escolha pelo formato de artigo ocorreu em função da qualidade desse tipo de publicação, que é submetido a um processo editorial que conta com a avaliação de pareceristas ad hoc para sua aprovação.

Conforme consta na Figura 1, inicialmente foram localizados 710 estudos, destes 153 foram excluídos por duplicata, 19 por estarem em outros idiomas, além do português, inglês e espanhol, e 18 por se constituírem em tipos distintos de publicação, como editoriais, resenhas e resumos de livros. A exclusão das duplicatas foi realizada de forma aleatória não privilegiando nenhuma base de dados específica. Em seguida, restaram 520 que foram analisados quanto a sua temática principal. Destes, 471 foram excluídos: 390 por não terem como temática principal o fracasso escolar, 48 por não envolverem a educação básica e 33 por serem estudos teóricos. Ao final, restaram 49 artigos, que tinham como foco o fracasso escolar, sendo que cinco deles não estavam na íntegra online de forma gratuita.

No que se refere às exclusões, destaca-se que a temática do fracasso escolar tem sido utilizada em diversas perspectivas. Ele é citado como uma consequência em artigos que discutem transtornos de déficit de atenção e hiperatividade, transtornos do espectro do autismo, distúrbios de conduta, depressão, epilepsia, bullying e $c y$ berbullying, bem como é relacionado ao uso da internet e do telefone celular entre adolescentes. Há também artigos sobre o uso de álcool e outras drogas e suicídio, que abordam o fracasso escolar como uma das suas possíveis causas. Artigos também foram excluídos por enfocar o risco de fracasso escolar em crianças devido à pobreza e do abandono, por viverem em orfanatos ou terem os pais aprisionados.

\section{Resultados}

A partir da análise dos artigos, foram selecionados 44 estudos que preencheram os critérios de inclusão. Tais artigos foram classificados tendo em vista o ano e o país de origem da publicação, o foco do estudo, o conceito de fracasso escolar considerado, os aspectos metodológicos (abordagem de pesquisa, participantes, instrumentos) e seus principais resultados.

No que diz respeito ao ano das publicações, houve certa instabilidade ao longo do tempo, conforme consta na Figura 2. Os anos de 2013, 2012 e 2010 se destacaram por contar com o maior número de publicações. Já o ano de 2014 e 2011 foram os que tiveram menor número de artigos. 


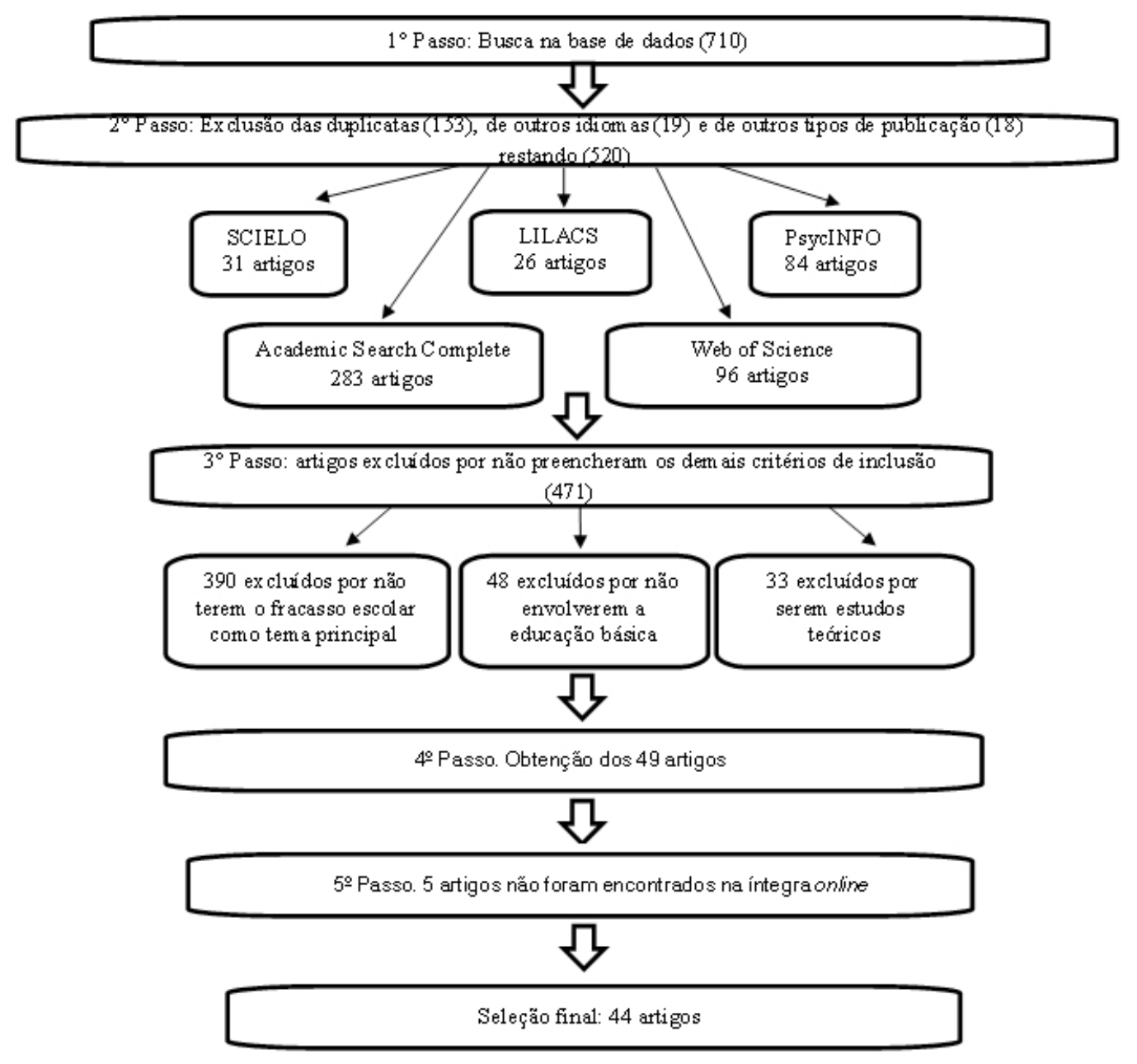

Figura 1. Procedimentos de seleção dos artigos.

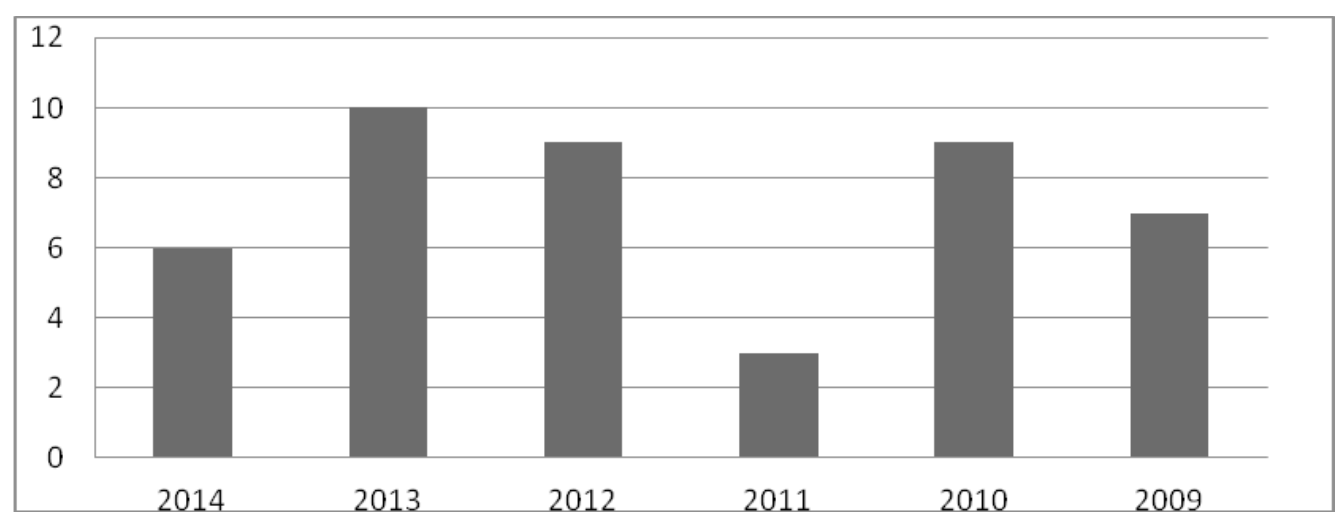

Figura 2. Número de publicações por ano.

Ao considerar o país de origem dos artigos, atenta-se para a sua diversidade. Foram 18 países diferentes, destacando-se o Brasil, os Estados Unidos, a Suécia, Portugal e a Espanha. Os estudos representavam quatro continentes diferentes, sendo que um deles envolveu participantes dos Estados Unidos e da Bulgária. A Figura 3 ilustra os países de origem dos estudos com o número de produções correspondente. 


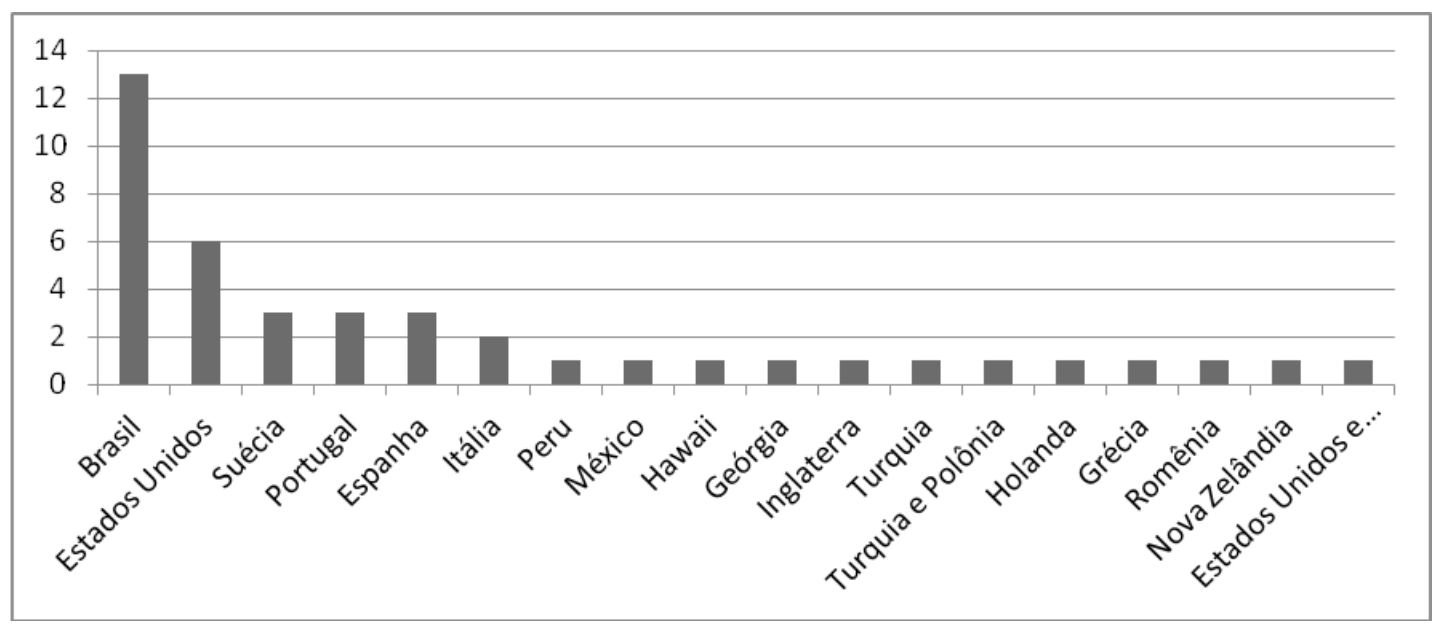

Figura 3. País de origem das publicações.

Para considerar o foco dos estudos, realizou-se uma análise de conteúdo (Bardin, 1977; Laville \& Dionne, 1999) a partir da qual derivaram seis categorias, a saber: (a) compreensão geral sobre o fracasso escolar, que contemplou 13 artigos que buscaram entender o fracasso escolar de forma ampliada e a partir de fontes de dados e/ou perspectivas de professores, alunos, pais e profissionais, sem nenhuma hipótese ou expectativa prévia (Bastos et al., 2009; Bray \& Leonardo, 2011; Ferrera, López, \& Rodríguez, 2014; Garcia \& Boruchovitch, 2014; Gomes \& Souza, 2009; Guler, 2013; Hancer, 2012; Hjörne \& Säljö, 2014; Jacomini, 2010; Kamal \& Bener, 2009; Lanza, Rhodes, Nix, Greenberg, \& Conduct Problems Prevention Research Group, 2010; Lynn, Bacon, Totten, Bridges, \& Jennings, 2010; Oliveira \& Macedo, 2011); (b) fracasso escolar relacionado aos alunos, que envolveu 13 artigos que consideraram variáveis que envolviam os próprios alunos (Bidjerano, 2010; Kamtsios \& Karagiannopoulou, 2013; Lopes \& Teixeira, 2012; Martinelli \& Genari, 2009; Millones, Leeuwen, \& Ghesquière, 2013; Odd, Evans, \& Emond, 2013; Pershey, 2010; Rombaldi et al., 2012; Sarver et al., 2012; Soares, Aranha, \& Antunes, 2013; Vasconcelos, 2010; Walkey, McClure, Meyer, \& Weir, 2013; Williams et al., 2013); (c) fracasso escolar relacionado à família, que abarcou três artigos com foco nas variáveis familiares (Berg, Rostila, Saarela, \& Hjern, 2014; Couto, 2010; Orgiles,
Johnson, Huedo-Medina, \& Espada, 2012); (d) fracasso escolar relacionado à escola, que considerou sete estudos em que aspectos da escola foram analisados (Lucio, Hunt, \& Bornovalova, 2012; Osti \& Brenelli, 2013; Paiva \& Del Prette, 2009; Soponaru, Tincu, \& Yorga, 2014; Spilt, Hughes, Wu, \& Kwok, 2012; Travi, Oliveira-Menegotto, \& Santos, 2009; Van den Bergh, Denessen, Hornstra, Voeten, \& Holland, 2010); (e) fracasso escolar relacionados aos aspectos sociais, a qual envolveu quatro artigos que tinham foco em aspectos da sociedade e da cultura (Corbi Gran \& Pérez, 2013; Dhavan, Stigler, Perry, Arora, \& Reddy, 2010; Herman, 2009; Patton, Woolley, \& Hong, 2011); e, (f) implicações do fracasso escolar, que agrupou quatro artigos que estudaram a relação entre fracasso escolar e consequências futuras, como uso de drogas, desenvolvimento de comportamentos delinquentes e depressi-vos e escolhas educacionais e profissionais (Gauffin,Vinnerljung, Fridell, Hesse, \& Hjern, 2013; Mocetti, 2012; Nocentini, Calamai, \& Menesini, 2012; Nunes, Pontes, Silva, \& Dell'Aglio, 2014). Essas categorias foram avaliadas por dois juízes que tiveram um percentual de concordância de $88,5 \%$.

Com relação ao conceito de fracasso escolar considerado em cada estudo, não houve uma padronização, cabendo aos autores definir o entendimento que tinham em cada contexto abordado. Constatou-se que tal conceito foi utilizado para se referir às notas baixas (Gauffin 
et al., 2013; Guler, 2013; Herman, 2009; Kamtsios \& Karagiannopoulou, 2013; Lucio et al., 2012; Odd et al., 2013; Orgiles et al., 2012; Soponaru et al., 2014; Spilt et al., 2012; Williams et al., 2013), à repetição de ano letivo (Corbi Gran \& Pérez, 2013; Dhavan et al., 2010; Ferrera et al., 2014; Jacomini, 2010; Kamal \& Bener 2009; Lopes \& Teixeira, 2012; Nocentini et al., 2012; Rombaldi et al., 2012; Soares et al., 2013), às dificuldades de aprendizagem e/ ou comportamentais (Bray \& Leonardo, 2011; Couto, 2010; Oliveira \& Macedo, 2011; Osti \& Brenelli, 2013; Travi et al., 2009; Vasconcelos, 2010), ao fato de os alunos terem abandonado o sistema de ensino antes do término da escolaridade obrigatória (Bastos et al., 2009; Berg et al., 2014; Mocetti, 2012), ou mesmo a partir da conceituação dos próprios alunos, ou seja, considerando o que eles entendiam como fracasso escolar (Bidjerano, 2010). Por fim, localizaram-se estudos que não apresentaram o conceito de fracasso escolar considerado (Garcia \& Boruchovitch, 2014; Gomes \& Souza, 2009; Hancer, 2012; Hjörne \& Säljö, 2014; Lanza et al., 2010; Lynn et al., 2010; Martinelli \& Genari, 2009; Millones et al., 2013; Nunes et al., 2014; Paiva \& Del Prette, 2009; Patton et al., 2011; Pershey, 2010; Sarver et al., 2012; Van den Bergh et al., 2010; Walkey et al., 2013).

Quanto aos aspectos metodológicos, no que se referiu à abordagem de pesquisa, $31 \mathrm{dos}$ artigos $(70,4 \%)$ localizados foram identificados como de abordagem quantitativa, enquanto que a abordagem qualitativa foi utilizada em 12 artigos $(27,3 \%)$ e a abordagem mista apenas em um estudo (2,3\%). Particularmente, entre os estudos brasileiros, dos 11 selecionados para revisão, oito utilizaram a abordagem qualitativa, quatro a quantitativa e um a mista. Em relação ao delineamento, 25 artigos se caracterizaram como transversais $(56,8 \%), 14$ como longitudinais $(31,8 \%)$, três como estudos de caso $(6,8 \%)$ e dois como etnografias $(4,6 \%)$.

No que diz respeito aos participantes, em 33 estudos os alunos foram a população analisada para compreensão do fracasso escolar (75\%), especialmente com a participação de adolescentes. Também participaram professores $(11,4 \%)$, famílias (9\%), equipe de saúde multidisciplinar da escola $(2,3 \%)$ e membros da escola juntamente com os alunos (2,3\%). Um aspecto relevante é que alguns estudos internacionais tinham grandes amostras. Por exemplo, os estudos realizados na Suécia, como o de Berg et al. (2014) e de Gauffin et al. (2013), contaram com 772.117 e com 1.405 .763 participantes, respectivamente. Ambos utilizaram o registro nacional do país (PIN - Personal Identification Number), em que constam dados integrados de saúde, educação e assistência social, desde o nascimento dos cidadãos.

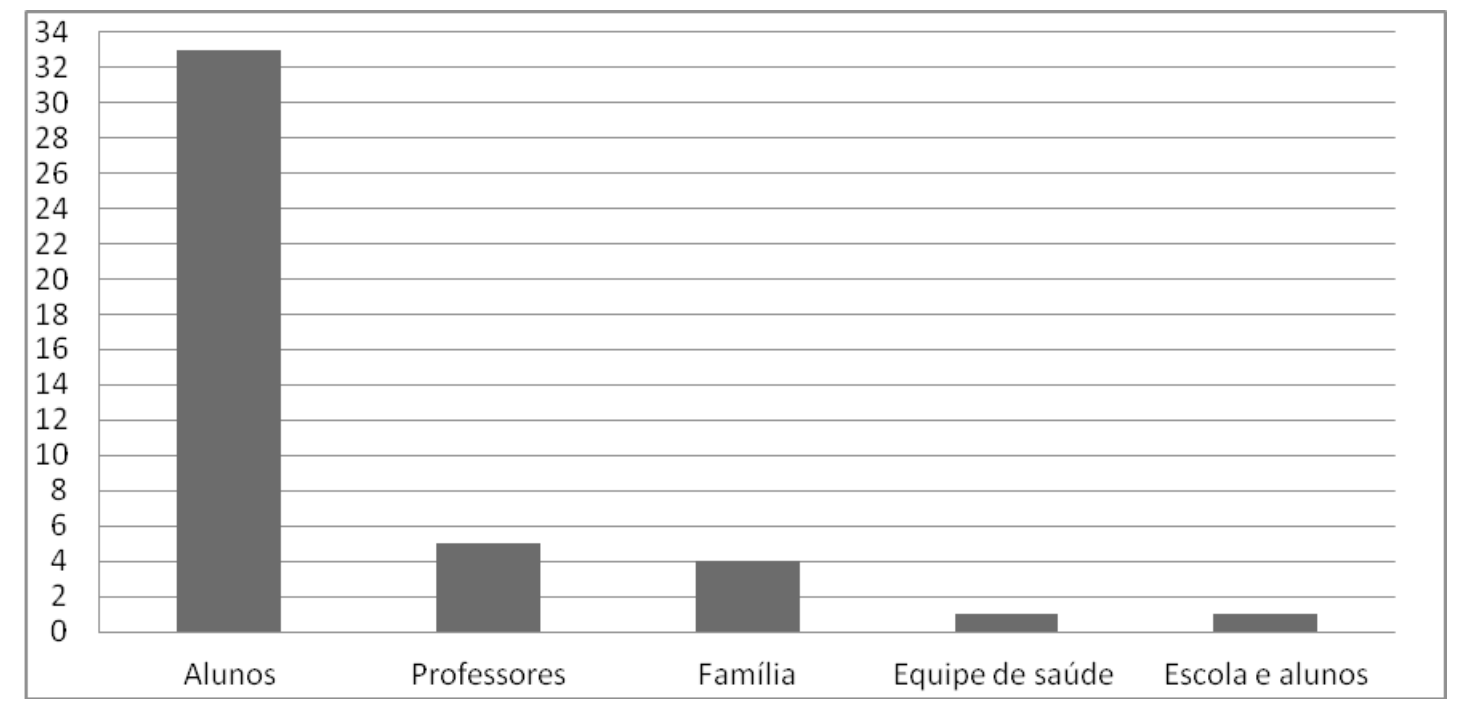

Figura 4. Participantes dos estudos. 
Referente aos instrumentos prevaleceu o uso de múltiplas técnicas de coleta de dados. Do total de 44 artigos, $16(36,4 \%)$ utilizaram apenas um instrumento, sendo que sete artigos propuseram o questionário, três realizaram entrevistas e três relataram intervenções clínicas. $\mathrm{O}$ grupo focal, o sistema conversacional e as reuniões da equipe de saúde da escola foram utilizados apenas em um artigo. Já 28 artigos $(63,6 \%)$ realizaram o processo de triangulação de dados (Barbour, 2009) através de distintos instrumentos, especialmente de escalas (autoestima, ansiedade, autoeficácia, ajustamento psicossocial etc), questionários (dados sociodemográficos, percurso escolar, uso de cigarro e outras drogas etc) ou testes padronizados (inteligência, a atenção, o desempenho escolar, problemas comportamentais etc), bem como fizeram uso de registros disponíveis em bancos de dados de outras pesquisas ou de sistemas educacionais, de saúde, de assistência social e de trabalho: 15 estudos utilizaram registros escolares e algum tipo de teste/escala/ questionário; oito usaram testes, questionários e escalas em conjunto; quatro consideraram diferentes tipos de registros dos alunos, como registros escolares, de assistência social, saúde e trabalho, um fez uso de observação, entrevista e grupo focal e, por fim, um utilizou observação e entrevista.

No tocante aos resultados dos artigos, eles foram agrupados de acordo com a categorização estabelecida a partir do foco pesquisado e não das conclusões obtidas. Como já referido, as categorias versaram sobre a compreensão geral sobre o fracasso escolar; o fracasso escolar relacionado aos alunos; o fracasso escolar relacionado à família; o fracasso escolar relacionado à escola; o fracasso escolar relacionado aos aspectos sociais; e as implicações do fracasso escolar. Grande parte das pesquisas analisou um recorte do fenômeno, apresentando separadamente diferentes aspectos sobre ele. Da igual forma, esses dados foram analisados por dois juízes que obtiveram um percentual de concordância de $92 \%$.

\section{Discussão}

A partir da presente revisão sistemática de literatura, constatou-se que o fracasso escolar é um fenômeno estudado mundialmente, pois nos últimos seis anos ele foi pesquisado em 18 países diferentes, considerando os idiomas selecionados neste estudo. Ao mesmo tempo, apesar da existência de diferenças organizacionais entre os sistemas de educação dos distintos países, pode-se verificar que as produções que investigaram esse fenômeno destacam-no como um problema para o sistema educacional local e também para os indivíduos que o vivenciam. Contudo, o número de estudos encontrados (44 artigos) pode ser considerado pequeno, diante da dimensão que o fracasso escolar ocupa de acordo com as estatísticas já relatadas (Unesco, 2014). Destaca-se que o Brasil foi o país que teve maior número de produções, o que demonstra a relevância desse assunto, pois ainda se evidenciam importantes índices de fracasso escolar (IBGE, 2010).

A dificuldade de pesquisar o fracasso escolar é evidenciada na definição de seu próprio conceito, pois não há consenso entre os autores. Como referido por Marchesi e Pérez (2004), o termo fracasso escolar é utilizado nos mais diferentes contextos, para distintas situações. Dentre os estudos avaliados, os principais conceitos utilizados foram reprovação, avaliação dos profissionais da escola (dificuldades de aprendizagem/ comportamentais) e escore obtido em determinados testes de desempenho. Entretanto, ressalta-se que estes, normalmente, avaliavam aptidões específicas de leitura, ortografia e matemática, desconsiderando outras habilidades também importantes, como motricidade, habilidade musical ou artística e capacidade de relação interpessoal. Esse dado revela a organização do atual sistema de ensino nacional e também internacional, que privilegia as capacidades acadêmicas e intelectuais. Também se questiona sobre o quanto o fracasso escolar pode trazer prejuízos para as pessoas que o vivenciam. A ideia que costuma prevalecer é a de que alguém fracassa (Marchesi \& Pérez, 2004) e, na grande maioria dos casos, 


\title{
Tabela 1
}

Resultados das Pesquisas Considerando o seu Foco

Foco dos

estudos

Resultados dos Estudos

\begin{abstract}
Culpabilização dos alunos pelo seu desempenho escolar (Bray \& Leonardo, 2011; Garcia
\& Boruchovitch, 2014; Gomes \& Souza, 2009; Guler, 2013; Hjörne \& Säljö, 2014)

Compreensão geral sobre o fracasso escolar

Questões familiares (Bastos et al., 2009; Oliveira \& Macedo, 2011), comunitárias (Lynn et al., 2010) e étnicas (Lanza et al., 2010) tiveram influência sob o fracasso escolar

Fracasso escolar entendido como a conjugação de fatores individuais, familiares e escolares (Ferrera et al., 2014; Hancer, 2012; Kamal \& Bener, 2009)

Reprovação considerada como algo que possibilitaria o aluno a alcançar um maior aprendizado
\end{abstract}

Fracasso escolar relacionado aos alunos

\section{Fracasso} escolar

relacionado à família

Fracasso escolar relacionado à escola

Fracasso escolar relacionado à aspectos sociais

Implicações do fracasso escolar (Jacomini, 2010)

Prematuridade entendida como preditora do fracasso escolar (Odd et al., 2013; Williams et al., 2013)

Habilidades de atenção associadas negativamente com o desempenho escolar (Sarver et al., 2012) Alunos com aspirações futuras tiveram um desempenho escolar melhor do que aqueles que não as possuíam (Walkey et al., 2013)

Alunos da quarta série, em contraponto com alunos de séries anteriores, manifestaram correlação positiva entre a motivação intrínseca para aprender e o desempenho escolar (Martinelli \& Genari, 2009) e alunos com baixo desempenho acadêmico viam-se mais negativamente do que seus pares (Millones et al., 2013)

Diante do fracasso escolar, os alunos manifestaram emoções negativas (Bidjerano, 2010); e expressaram maior número de avaliações negativas sobre o seu desempenho, de modo que os seus projetos de vida eram menos ambiciosos (Lopes \& Teixeira, 2012)

As notas ruins influenciaram o estado emocional (Kamtsios \& Karagiannopoulou, 2013) e colocavam os alunos em risco de abandono escolar (Pershey, 2010)

Fracasso escolar foi relacionado à estrutura individual e familiar, num caso em que o menino queria aprender, mas, em seu tempo (Vasconcelos, 2010)

Adolescentes que praticavam mais atividade física aos 11 anos tiveram maior probabilidade de ter fracasso escolar (Rombaldi et al., 2012); e o tipo de modalidade esportiva teve relação com o fenômeno, de maneira que os alunos que praticavam futsal ou futebol tinham maior índice de fracasso escolar em comparação com aqueles que praticavam natação e ginástica (Soares et al., 2013)

Fracasso escolar entendido como um sintoma familiar (Couto, 2010)

Separação dos pais (Orgiles, et al., 2012) e morte dos pais na infância (Berg et al., 2014) tiveram relação com o fracasso escolar

Fracasso escolar relacionado a dificuldades no relacionamento professor-aluno (Osti \& Brenelli, 2013; Spilt et al., 2012), a maneira como o aluno é percebido pelos colegas (Soponaru et al., 2014), as atitudes dos professores (Paiva \& Del Prette, 2009; Van den Bergh et al., 2010) ou à organização institucional da escola (Lucio et al., 2012; Travi et al., 2009)

Uso de tabaco como preditor do fracasso escolar (Corbi Gran \& Pérez, 2013; Dhavan et al., 2010) Fracasso escolar relacionado à exposição à violência (Patton et al., 2011) e discriminação étnica (Herman, 2009)

Fracasso escolar associado ao risco de uso de drogas ilícitas na juventude independentemente do nível socioeconômico (Gauffin et al., 2013); aumento de comportamentos delinquentes e depressivos (Nocentini et al., 2012); baixas expectativas de futuro acadêmico (Nunes et al., 2014) Fracasso escolar afetou as escolhas educacionais e profissionais futuras (Mocetti, 2012) 
se entende que esse alguém é o aluno, como fica evidenciado nos resultados dos estudos.

Outra questão importante e discutida em muitos dos estudos analisados se refere às causas e implicações do fracasso escolar. Com esse objetivo alguns autores partiram da compreensão do fenômeno como um todo e outros de aspectos específicos, como os individuais (aspecto biológico, história de vida, motivação, atividade esportiva), os familiares (separação dos pais, morte dos pais na infầncia, escolaridade da mãe, estrutura familiar), os escolares (relação professor-aluno, questões institucionais) e os sociais (abuso de drogas, violência, nível socioeconômico), além daqueles que avaliaram as implicações futuras desse fenômeno para os envolvidos.

Quanto aos aspectos metodológicos destaca-se a prevalência de estudos de abordagem quantitativa sob as qualitativas, especialmente em nível internacional. Já no Brasil, particularmente, predominou o uso da abordagem qualitativa. Constata-se, portanto, que nacionalmente há uma tendência oposta a dos estudos internacionais quanto à abordagem de pesquisa, o que pode estar relacionado à ausência de bancos de dados integrados entre as diferentes áreas do saber, bem como a carência de registros longitudinais sobre saúde e educação.

No que se refere ao delineamento, ressalta-se a pluralidade de desenhos de pesquisa. Nessa perspectiva, pontua-se como positiva a realização de estudos longitudinais, os quais possibilitam analisar mudanças que ocorrem ao longo do tempo em determinadas variáveis e contextos, bem como, entre suas relações, possibilitando realizar inferências sobre a sua evolução, causas e efeitos (Sampieri, Callado, \& Lucio, 2013).

No tocante aos participantes dos estudos analisados, constatou-se que a maioria deles parte de variáveis relacionadas aos próprios alunos e utiliza-se dessa população para entender o fracasso escolar. Destaca-se o tamanho das amostras nos estudos internacionais, especialmente nos da Suécia, país onde cada indivíduo possui um número de cadastro unificado, com registros de saúde, educação e assistência social desde o nascimento, possibilitando o fácil acesso a dados para pesquisas. Também cabe salientar os estu- dos de Herman (2009), Lucio et al., (2012), Mocetti (2012), Odd et al., (2013), Rombaldi et al., (2012), Walkey et al., (2013) e Williams et al., (2013), que foram compostos por mais de 4.000 participantes. Entretanto, sabe-se que o tamanho da amostra não garante a qualidade metodológica do estudo.

Muitos estudos, em especial os de abordagem quantitativa, utilizaram a triangulação de dados o que possibilita confirmar ou contradizer os resultados obtidos entre os participantes de diferentes modos (Barbour, 2009). Tais estudos adotaram mais de um instrumento, fazendo uso de registros escolares, questionários, escalas e testes padronizados para obtenção dos dados para pesquisa. Já dentre os estudos de abordagem qualitativa, predominou o uso de um único instrumento.

Destaca-se que os resultados encontrados nos estudos analisados são relevantes para o meio científico, ao mesmo tempo em que revelam uma perspectiva histórica que continua vigente na busca da compreensão do fracasso escolar: isola-se o contexto e busca-se entender as variáveis de forma independente. Além disso, as variáveis vinculadas aos alunos são enfatizadas, o que pode estar relacionado ao fato de eles terem sido os participantes da grande maioria das pesquisas selecionadas. Foram poucos os estudos que investigaram, concomitantemente, os aspectos individuais, familiares, escolares e sociais para o entendimento desta temática.

De forma semelhante, a exceção do trabalho de Hjörne e Säljö (2014), os demais estudos analisados não buscaram entender como os profissionais da educação e saúde que atuam nas escolas, por exemplo, entendem e abordam o fracasso escolar. Portanto, sugere-se a realização de pesquisas que busquem compreender como os acadêmicos de pedagogia, psicologia e demais cursos das áreas citadas compreendem a temática, visto que são eles que conduzirão e trabalharão com os alunos no futuro.

Apesar do reconhecimento do problema apresentado, ficou explícito que todos os estudos problematizaram o fracasso escolar como um fenômeno preocupante, que pode impactar no aluno, sua família, escola e sociedade. O estu- 
do de Lucio et al. (2012), que buscou identificar um número mínimo de fatores que colocariam alunos em fracasso, destacando aspectos como o engajamento e as expectativas acadêmicas e a relação professor-aluno, concluiu que não era a natureza do fator, mas o número de fatores que configuraria o fracasso escolar, reafirmando a complexidade e multideterminação do fenômeno (Lahire, 2004; Ullastres, 2003). Nessa mesma perspectiva, Ferrera et al. (2014), Hancer (2012) e Kamal e Bener (2009) concluíram pela conjugação de fatores individuais, familiares e escolares para a incidência de sucessivas reprovações.

Ao considerar o contexto nacional, sabe-se que a reprovação está na própria origem da escola brasileira e, por isso, tende a ser naturalizada (Ribeiro, 1991). De forma semelhante, afeta a grande maioria dos países do mundo (Torres, 2004). Contudo, quando se analisa sobre em quais realidades a reprovação mais incide, retorna-se a tese da carência cultural (Angelucci et al., 2004), que sustenta que os mais desfavorecidos socioeconomicamente seriam os mais afetados e, por isso, o público mais estudado. Diante do exposto, conclui-se pela necessidade de revisar as políticas educacionais, visto a necessidade de reversão e/ou prevenção dessa realidade educacional, a qual deverá ser feita baseada em evidências.

\section{Conclusões}

A presente revisão da literatura buscou sistematizar o que as pesquisas atuais têm considerado ao trabalhar com a temática do fracasso escolar na educação básica. Os estudos analisados representam uma fonte importante de conhecimento para os pesquisadores da área, bem como para os profissionais da psicologia e da educação que trabalham com crianças e adolescentes. Também revelam a perspectiva a partir da qual esse fenômeno vem sendo analisado nacional e internacionalmente.

Ao considerar o número de publicações sobre a temática, constata-se o quanto o fracasso escolar é relevante e estudado a partir de diferentes perspectivas. Entretanto, atualmente ele não tem sido a temática central dos estudos, mas é abordado como causa ou consequência de fenômenos como o uso de álcool, cigarro e outras drogas, o bullying ou ciberbullying, a epilepsia, entre outros.

Ressalta-se que mesmo após tantos anos de estudo dessa temática, ainda se continua a culpabilizar de modo particular os alunos, suas famílias ou seus professores e/ou a escola pelo fracasso escolar, quando na verdade tais dimensões precisam ser consideradas de forma conjunta diante da complexidade do fenômeno. É importante salientar que, ao considerar o aluno, as pesquisas também se preocuparam com os seus aspectos psicológicos, especialmente com o impacto que o fracasso escolar pode ter na sua autoestima e autoconfiança, destacando a necessidade de considerar o fenômeno pelo viés da psicologia clínica, evidenciando que ele pode trazer consequências importantes para os indivíduos. Trata-se de uma situação sócio-histórica que se manifesta e traz consequências para todas as sociedades, como demonstrado nos diferentes países que o estudaram.

Convém destacar que a amostra de estudos analisada é apenas um recorte das pesquisas sobre o fracasso escolar na educação básica, considerando as bases de dados selecionadas, os descritores e os critérios de seleção utilizados nesta revisão. Sabe-se que alguns estudos também poderiam ser categorizados quanto ao objetivo, às variáveis examinadas e aos resultados de outras formas, mas optou-se por escolher a categoria a qual o estudo melhor correspondia a partir dos critérios da análise de conteúdo realizada. Para pesquisas futuras sugere-se a consulta a outras bases científicas e a inserção de outros termos que são utilizados como sinônimos de fracasso escolar e foram identificados nos estudos revisados, como, por exemplo, "baixo desempenho escolar" ou "abandono escolar", além de considerar um período maior de tempo das publicações.

Por fim, destaca-se a importância de intervir junto as famílias e comunidade escolar, face a complexidade do fracasso escolar, buscando modificar discursos, especialmente aqueles que ainda centram o problema no aluno e que partem de dentro da própria escola. Na medida em 
que o fracasso escolar persiste geram-se novos fracassos, como, por exemplo, dos alunos que desanimam e desistem de estudar, das escolhas pessoais e profissionais posteriores que fazem, entre tantas outras questões. Claramente, não é uma regra, mas faz-se importante estar atento para as possíveis marcas que o fracasso escolar pode imprimir naqueles que o vivenciam, tendo em vista que a sociedade atual reconhece a importância da escolarização para a inserção social e no mercado de trabalho.

\section{Referências}

Angelucci, C. B., Kalmus, J., Paparelli, R., \& Patto, M. H. S. (2004). O estado da arte da pesquisa sobre o fracasso escolar (1991-2002): Um estudo introdutório. Educação e Pesquisa, 30(1), 51-72. doi:10.1590/S1517-97022004000100004

Asbahr, F. S. F., \& Lopes, J. S. (2006). A culpa é sua. Psicologia USP, 17(1), 53-73. doi:10.1590/ S0103-65642006000100005

Barbour, R. (2009). Grupos focais (M. F. Duarte, Trad.). Porto Alegre, RS: Artmed.

Bardin, L. (1977). Análise de conteúdo (L. Reto \& A. Pinheiro, Trads.). São Paulo, SP: Edições 70.

Bastos, A., Fernandes, G. L., \& Passos, J. (2009). Analysis of school failure based on Portuguese micro data. Applied Economics Letters, 16, 1639-1643. doi:10.1080/13504850701604094

Berg, L., Rostila, M., Saarela, J., \& Hjern, H. (2014). Parental death during childhood and subsequent school performance. Pediatrics, 133(4), 681689. doi:10.1542/peds.2013-2771

Bidjerano, T. (2010). Self-conscious emotions in response to perceived failure: A structural equation model. The Journalof Experimental Education, 78, 318-342. doi:10.1080/00220970903548079

Bray, C. T., \& Leonardo, N. S. T. (2011). As queixas escolares na compreensão de educadoras de escola pública e privada. Revista Semestral da Associação Brasileira de Psicologia Escolar e Educacional, 15(2), 251-261. doi:10.1590/ S1413-85572011000200007

Corbi Gran, B., \& Pérez, M. A., Nieto (2013). El efecto del absentismo y el fracaso escolar enel consumo de tabaco, en una muestra de estudiantes de $3^{\circ}$ y $4^{\circ}$ de la educación secundaria obligatoria. Health and Addictions, 13(1), 53-58.
Couto, M. P. (2010). As novas organizações familiares e o fracasso escolar. Psicologia da Educação, 30, 57-66.

Delors, J. (1998). Educação um tesouro a descobrir: Relatório para UNESCO da Comissão Internacional sobre Educação para o século XXI. Recuperado em http://dhnet.org.br/dados/ relatorios/a_pdf/r_unesco_educ tesouro_descobrir.pdf

Dhavan, P., Stigler, M. H., Perry, C. L., Arora, M., $\&$ Reddy, K. S. (2010). Is tobacco use associated with academic failure among government school students in urban India? Journal of School, 80(11), 552-560. doi:10.1111/j.17461561.2010.00541.x

Ferrera, J. M. C., López, C. M., \& Rodríguez, R. S. (2014). La repetición de curso y sus factores condicionantes em España. Revista de Educación, 365, 12-37. doi:10.4438/1988-592XRE-2014-365-263

Garcia, N. R., \& Boruchovitch, E. (2014). Atribuições de causalidade para o desempenho escolar e resiliência em estudantes. Psico-USF, 19(2), 277286. doi:10.1590/1413-82712014019002003

Gauffin, K., Vinnerljung, B., Fridell, M., Hesse, M., \& Hjern, A. (2013). Childhood socio-economic status, school failure and drug abuse: A Swedish National Cohort Study. Addiction, 108(8), 14411449. doi:10.1111/add.12169

Gomes, C., \& Souza, V. L. T. (2009). Fracassos, representações e exclusões no processo de permanência na escola. Revista de Psicopedagogia, 26(79), 41-47.

Guler, M. P. D. (2013). Success and failure in science education: A focus group study on Turkish students. Journal of Baltic Science Education, 12(6), 716-729.

Hancer, A. H. (2012). Reasons of academic failure in Turkish and Polish $6^{\text {th }}$ grade primary school students. International Journal of Academic Research - Part B, 4(4), 78-82. doi:10.7813/2075$-4124.2012 / 4-4 / B .11$

Herman, M. R. (2009). The Black-White-Other Achievement Gap: Testing theories of academic performance among multiracial and monoracial adolescents. Sociology of Education, 82(1), 2046. doi:10.1177/003804070908200102

Hjörne, E., \& Säljö, R. (2014). Analysing and preventing school failure: Exploring the role of multi-professionality in pupil health team meetings. 
International Journal of Educational Research 63, 5-14. doi:10.1016/j.ijer.2012.09.005

Instituto Brasileiro de Geografia e Estatística. (2010). Censo demográfico de 2010. Recuperado em http://seriesestatisticas.ibge.gov.br/lista_tema. aspx?op $=0 \& n o=4$

Jacomini, M. A. (2010). Por que a maioria dos pais e alunos defende a reprovação? Cadernos de Pesquisa, 40(141), 895-919.

Kamal, M., \& Bener, A. (2009). Factors contributing to school failure among school children in a very fast developing Arabian society. Oman Medical Journal, 24(3), 212-217. doi:10.5001/ omj.2009.42

Kamtsios, S., \& Karagiannopoulou, E. (2013). Conceptualizing students' academic hardiness dimensions: A qualitative study. European Journal Psychology Education, 28, 807-823. doi:10.1007/s10212-012-0141-6

Lagana-Riordan, C., Aguilar, J., Franklin, C., Streeter, C., Kim, J., Tripodi, S., \& Hopson, L. (2011). At-risk students's perceptions of traditional schools and a solutions-focused public alternative school. Preventing School Failure, 55(3), 105-114. doi:10.1080/10459880903472843

Lahire, B. (2004). As origens da desigualdade escolar (E. Rosa, Trad.). In A. Marchesi \& C. H. Gil (Eds.), Fracasso escolar: Uma perspectiva multicultural (pp.69-75). Porto Alegre, RS: Artmed.

Lanza, S. T., Rhodes, B. T., Nix, R. L., Greenberg, M. T., \& Conduct Problems Prevention Research Group. (2010). Modeling the interplay of multilevel risk factors for future academic and behavior problems: A person-centered approach. Development and Psychopathology, 22, 313-335. doi:10.1017/S0954579410000088

Laville, C., \& Dionne, J. (1999). A construção do saber: Manual de metodologia da pesquisa em ciências humanas (L. M. Siman, Trad.). Porto Alegre, RS: Artes Médicas.

Lei 12.796 de 4 de abril de 2013. (2013). Altera a Lei número 9.394 de 20 de dezembro de 1996.

Lei de Diretrizes e Bases da Educação Nacional. (1996). Lei 9.394 de 20 de dezembro de 1996.

Lopes, A. R., \& Teixeira, M. O. (2012). Projetos de carreira, autoeficácia e sucesso escolar em ambiente multicultural. Revista Brasileira de Orientação Profissional, 13(1), 7-14.
Lucio, R., Hunt, E., \& Bornovalova, M. (2012). Identifying the necessary and sufficient number of risk factors for predicting academic failure. Developmental Psychology, 48(2), 422-428. doi:10.1037/a0025939

Lynn, M., Bacon, J. N., Totten, T. L., Bridges, T. L., III, \& Jennings, M. (2010). Examining teachers' beliefs about African American male students in a low-performing high school in an African American school district. Teachers College Record, 112(1), 289-330.

Marchesi, A., \& Pérez, E. M. (2004). A compreensão do fracasso escolar. In A. Marchesi \& C. H. Gil (Eds.), Fracasso escolar: Uma perspectiva multicultural (E. Rosa, Trad., pp.17-33). Porto Alegre, RS: Artmed.

Marshall, J. H. (2003). Grade repetition in Honduran primary schools. International Journal of Educational Development, 23, 591-605. doi:10.1016/S0738-0593(03)00060-9

Martinelli, S. C., \& Genari, H. M. (2009). Relações entre desempenho escolar e orientações motivacionais. Estudos de Psicologia (Natal), 14(1), 13-21. doi:10.1590/S1413294X2009000100003

Millones, D., Leeuwen, K., \& Ghesquière, P. (2013). Associations between psychosocial functioning and academic achievement: The Peruvian case. Universitas Psychologica, 12 (3), 725-737. doi:10.11144/Javeriana.UPSY12-3.apfa

Mocetti, S. (2012). Educational choices and the selection process: Before and after compulsory schooling. Education Economics, 20(2), 189209. doi:10.1080/09645291003726434

Nocentini, A., Calamais, G., \& Menesini, E. (2012) Codevelopment of delinquent and depressive symptoms across adolescence: Time-invariant and time-varying effects of school and social failure. Journal of Clinical Child \& Adolescent Psychology, 41(6), 746-759. doi:10.1080/15374 416.2012.728155

Nunes, T. G., Pontes, F. A. R., Silva, L. I. C., \& Dell'Aglio, D. D. (2014). Fatores de risco e proteção na escola: Reprovação e expectativas de futuro de jovens paraenses. Revista Quadrimestral da Associação Brasileira de Psicologia Escolar e Educacional, 18(2), 203-210. doi:10.1590/2175$3539 / 2014 / 0182732$

Odd, D., Evans, D., \& Emond, A. (2013). Preterm birth, age at school entry and educational 
performance. PlosOne, 8(10), 1-6. doi:10.1371/ journal.pone.0076615

Oliveira, F. N., \& Macedo, L. (2011). Resiliência e insucesso escolar: Uma reflexão sobre ás salas de aprendizagem. Estudos e Pesquisas em Psicologia, 11(3), 983-1004.

Organização das Nações Unidas. (1948). Declaração Universal dos Direitos Humanos. Recuperado em www.direitoshumanos.usp.br

Organização das Nações Unidas para a Educação, Ciência e Cultura. (1990) Declaração mundial sobre educação para todos: Satisfação das necessidades básicas de aprendizagem. Jomtien, Thailand: Author.

Organização das Nações Unidas para a Educação, Ciência e Cultura. (2000). Educação para todos: O compromisso de Dakar. Recuperado em http://unesdoc.unesco.org/ images/0012/001275/127509porb.pdf

Organização das Nações Unidas para a Educação, Ciência e Cultura. (2014). Relatório do monitoramento global de EPT 2013/4 - Ensinar e aprender: Alcançar a qualidade para todos. Recuperado em http://unesdoc.unesco.org/ images/0022/002256/225654POR.pdf

Organização para a Cooperação e o Desenvolvimento Econômico. (1998). Overcoming failure at school. Paris: Author.

Organização para a Cooperação e o Desenvolvimento Econômico. (2010). Overcoming school failure: Policies that work. Retrieved from http://www. oecd.org/education/school/45171670.pdf

Organização para a Cooperação e o Desenvolvimento Econômico. (2012). Equality and Quality in Education. Supporting Disavantaged Students and Schools. doi:10.1787/9789264130852-en

Orgiles, M., Johnson, B. T., Huedo-Medina, T. B., \& Espada, J. P. (2012). Self-concept and social anxiety as predictor variables of academic performance of Spanish adolescents with divorced parents. Eletronic Journal of Research in Educational Psychology, 10(1), 57-72.

Osti, A., \& Brenelli, R. P. (2013). Sentimentos de quem fracassa na escola: Análise das representações de alunos com dificuldades de aprendizagem. Psico-USF, 18(3), 417-426. doi:10.1590/ S1413-82712013000300008

Paiva, M. L. M. F., \& Del Prette, Z. A. P. (2009). Crenças docentes e implicações para o processo de ensino-aprendizagem. Revista Semestral da Associação Brasileira de Psicologia Escolar e Educacional, 13(1), 75-85.

Patton, D. U., Woolley, M. E., \& Hong, J. S. (2011). Exposure to violence, student fear, and low academic achievement: African American males in the critical transition to high school. Children and Youth Services Review, 34(2), 388-395. doi:10.1016/j.childyouth.2011.11.009

Pershey, M. G. (2010). A comparison of African American students' self-perceptions of school competence with their performance on state-mandated achievement tests and normed tests of oral and written language and reading. Preventing School Failure, 55(1), 53-62. doi:10.1080/10459880903472835

Ribeiro, S. C. (1991). A pedagogia da repetência. Estudos Avançados, 12(5), 7-21.

Rombaldi, A. J., Clark, V. L., Reichert, F. F., Araújo, C. L. P., Assunção, M. C., Menezes, A. M. B., ...Hallal, P. C. (2012). Incidence of school failure according to Baseline Leisure-Time Physical Activity Practice: Prospective study. Journal of Adolescent Health, 51(Suppl.), S22-S26. doi:10.1016/j.jadohealth.2012.06.024

Rumberger, R. W. (2011). Introdution. In R. W. Rumberger, Droping out (pp. 1-19). Retrieved from http://education.ucsb.edu/rumberger/book/ ch1.pdf

Sampieri, R. H., Callado, C. F., \& Lucio, M. P. B. (2013). Metodologia de pesquisa. Porto Alegre, RS: Penso.

Sarver, D., Rapport, M. D., Kofler, M. J., Scanlan, S., Raiker, J., \& Altro, T. A., \& Bolden, J. (2012). Attention problems, phonological short-term memory, and visuospatial short-term memory: Differential effects on near- and long-term scholastic achievement. Learning and Individual Differences 22, 8-19. doi:10.1016/j.lindif.2011.09.010

Soares, J. P., Aranha, A. M., \& Antunes, H. L. (2013) Relação entre os setores de prática desportiva, as modalidades desportivas e o aproveitamento escolar. Motricidade, 9(3), 3-11.

Soponaru, C., Tincu, C., \& Iorga, M. (2014). The influence of the sociometric status of students on academic achievement. Agathos: An International Review of the Humanities and Social Sciences, 5(2), 149-168. 
Spilt, J. L., Hughes, J. N., Wu, J. Y., \& Kwok, O. (2012). Dynamics of teacher-student relationships: Stability and change across elementary school and the influence on children's academic success. Child Development, 83(4), 1180-1195. doi:10.1111/j.1467-8624.2012.01761.x

Travi, M. G. G., Oliveira-Menegotto, L. M., \& Santos, G. A. (2009). A escola contemporânea diante do fracasso escolar. Revista Psicopedagogia, 26(81), 425-434.

Torres, R. M. (2004). Repetência escolar: Falha do aluno ou falha do sistema? In A. Marchesi \& C. H. Gil (Eds.), Fracasso escolar: Uma perspectiva multicultural (E. Rosa, Trad., pp. 34-47). Porto Alegre, RS: Artmed.

Ullastres, A. M. (2003). El fracaso escolar em ESpaña. Madrid: FundaciónAlternativas.

Van den Bergh, L., Denessen, E., Hornstra, L., Voeten, M., \& Holland, R. (2010). The implicit prejudiced attitudes of teachers: Relations to teacher expectations and the ethnic achievement gap. American Educational Research Journal, 47(2),497-527. doi:10.3102/0002831209353594

Vasconcelos, M. A. M. (2010). A dimensão do fracasso escolar na vertente da clínica psicanalítica: O caso do pequeno Roberto. Pesquisas e Práticas Psicossociais, 4(2), 238-242.

Vieira, M. F. A., Matijasevich, A., Damiani, M. F., Madruga, S. W., Neutzling, M. B., Menezes, A. M. B., \& Hallal, P. C. (2012). Prevalência de retenção escolar e fatores associados em adolescentes da coorte de nascimentos de $1993 \mathrm{em}$ Pelotas, Brasil. Revista Panamericana Salud Pública, 31(4), 303-309. doi:10.1590/S102049892012000400006
Walkey, F. H., McClure, J., Meyer, L. H., \& Weir, K. F. (2013). Low expectations equal no expectations: Aspirations, motivation, and achievement in secondary school. Contemporary Educacional Psychology, 38, 306-315. doi:10.1016/j.cedpsych.2013.06.004

Williams, B. L., Dunlop, A. L., Kramer M., Dever, B. V., Hogue, C., \& Jain, L. (2013). Maternal factors perinatal origins of first-grade academic failure: Role of prematurity and maternal factors. Pediatrics, 131(4), 693-700. doi:10.1542/ peds.2012-1408

Zago, N. (2011). Fracasso e sucesso escolar no contexto das relações família e escola: Questionamentos e tendências em sociologia da educação. Revista Luso-Brasileira, 2(3), 57-83.

Zucoloto, P. C. S. V., \& Patto, M. H. S. (2007). O médico higienista na escola: As origens históricas da medicalização do fracasso escolar. Revista Brasileira de Crescimento e Desenvolvimento Humano, 17(1), 136-145.

Recebido: 05/06/2015

$1^{a}$ revisão: $15 / 12 / 2015$

Aceite final: 04/01/2016 